Special Issue: Social Cognition and Self. Citation: El Moghrabi, H. (2015). Acceptance in blame: How and why we blame the victims of street harassment. Behavioural Sciences Undergraduate Journal, 2(1), 74-83.

Author: Hanaa El Moghrabi, Psychology, Mount Royal University (MRU)

Correspondence: hanaaelmoghrabi@gmail.com

Reviewers: Alain Morin, MRU; Kelsie Moore, MRU; Mabel Yu, MRU

Editor: Famira Racy, MRU

Acknowledgements: The BSUJ thanks Dr. Dave Mumby of Concordia University for Associate Editorial contributions to this article.

Copyright: (C) 2015 Hanaa El Moghrabi. This is an open-access article distributed under the terms of the Creative Commons Attribution 4.0 International License. The use, distribution or reproduction of this article in other forums is permitted, provided the original author(s) or licensor are credited, a link to the licence is provided (CC for 4.0), it is indicated whether or not changes were made to the original article, and other conditions of the BSUJ and the other forums are met.

\title{
Acceptance in Blame: How and why we Blame the Victims of Street Harassment
}

\begin{abstract}
Globally, and on a daily basis, women are subjected to unwanted verbal and/or physical intrusions such as catcalling, leering, honking, sexually explicit or sexist comments, touching or grabbing, amongst other actions that are all considered street harassment. This paper is a review of some of the literature available, which focuses on the psychological and feminist aspects of street harassment and victim blaming through social, cognitive, intersectional, and economic lenses. Regarding psychological theories, I will examine reasons why victim blaming happens through the theories of the just-world hypothesis, cognitive dissonance, and the bystander effect. The feminist theories touch on the basics of objectification and power dynamics found within gender, which can help us understand why street harassment happens. Lastly, I will emphasize the importance of starting a conversation about the pervasiveness of street harassment and victim blaming, and why it is important to know where the blame should be instead of where it is almost always placed.
\end{abstract}

Keywords: street harassment, victim blaming, gender-based violence, cognitive effects, feminist theories, just-world, cognitive dissonance, group-think

\section{Introduction}

Street harassment is as pervasive as it is abhorrent and its consequences not only stigmatize the victims but also leave many of the perpetrators unscathed by the law. Regardless of gender, race, age, ability and other variables, people are subjected to daily street harassment by strangers around the world (Hollaback Ottawa, 2014). This makes travelling from point A to point B incredibly difficult for these people as they are often followed, catcalled, leered at, grabbed, or touched. All of these behaviours are unwarranted and not asked for in any shape or form. Further, such behaviour causes the victim to feel unsafe in any environment (Fairchild, 2010). The victims of street harassment tend to be disproportionately blamed rather than the harassers themselves. As in many societies, the victims were supposedly doing something wrong (being blamed), whether they are walking by themselves, travelling at night, wearing something 
too tight or short or colourful, or generally generating too much attention to themselves (Stop Street Harassment National Survey, 2014). It is important to highlight what street harassment is, what victim blaming is, and how this can be stopped.

Street harassment is unwanted advances, or verbal and/or physical intrusions that cause the target - the victim — to feel unsafe (Fairchild \& Rudman, 2008). The pervasiveness of street harassment tends not to be seen as detrimental; in fact, it is just seen as complimenting the passerby. But it is not as simple as that; these seemingly harmless comments feed into a power dynamic that is seen globally, in which one party has more power over the other party, and thus the party which has the most power tends to exercise this abuse to the extent that the victim is treated as though they are not human, but an object (Gannon, 2005). Perpetuating ideas that men are more powerful over women causes a discrepancy in cognition that excludes the idea that men and women are equal, but instead that men are more important than women. Street harassment is gender-based violence, albeit a more insidious one that has not always been an important topic in everyday life. Indeed, Fairchild \& Rudman (2008) contend against the idea that street harassment is not an important topic, stating that street harassment has long been ignored by the fields of social sciences and in most feminist literature, but is in dire need of more research and awareness.

The lack of attention over the years has led to a normalization of street harassment up until recent years when several individuals have spoken up to spread awareness. Normalization is when something has become so conditioned to be commonplace and completely desensitized in a society that it is defined as normal-not deviant-behaviour (Foucault, 1975). In this case, street harassment is seen as normal behaviour because it has become so commonplace that it has not been seen as problematic behaviour, until recent years with rising awareness. According to the Stop Street Harassment National Survey (2014), between 27\% - 99\% of individuals who took their survey $(n=2040)$ were victims of one or more of the following: leering, honking and whistling, sexist or sexually explicit comment(s), making vulgar gestures, kissing noises, following, blocking paths, sexual touching or grabbing, masturbating, and assaulting, which all constitutes misogyny. In light of this problem, I will highlight both psychological and feminist theories in a social, cognitive, and economic context to illuminate the causes and possible preventions of street harassment and victim blaming.

\section{Psychological Theories}

Social cognition plays a large part in street harassment and victim blaming insomuch that social perceptions such as attitudes, schemas, and inferences, affect how individuals act towards others (Fiske \& Taylor, 1991; Morin, 2014). The perpetrators will act in a particular way when they are harassing women (e.g., based on past experiences, heuristics, gender expectations), and the general public will act in a particular way when dealing with the victim. For example, both perpetrators and the public may have attitudes toward what the victim was wearing and when/where the victim was walking, or may make use of social scripts perpetuating that the victim is to blame because the victim got what the victim deserved.

Biased perceptions and attitudes about the harassment are major factors in victim blaming, such as believing that bad things happen to bad people (Just-world hypothesis; e.g., van de Boss \& Mass, 2009), or shifting blame from the perpetrator to the victim because the victim must be at fault for his/her actions (victim blaming). These perceptions and attitudes are typically conditioned responses of stereotypical gender roles, bombardment of skewed media, diffusion of 
responsibility, and creating cognitive harmony when cognition becomes erratic (Bennett, Banyard, \& Garnhart, 2014).

\subsection{Just-world Hypothesis, Cognitive Dissonance \& Self-blame}

The just-world hypothesis is a type of survival mechanism that allows the observers of an attack to blame the victim instead of the perpetrator, thus invoking a type of false-sense of security so the observers will feel safe (Lerner, 1966). By blaming the victim, the observer will feel that if the victim did not perform certain actions he/she would have not been harassed in the first place. Actions such as not walking around at night, walking alone, or wearing something 'attention-grabbing' justify the observer blaming the victim because of erroneous ideas, for example that there are people who are dangerous, have ill-intent, and will act out on anyone during any time of day. By using this survival mechanism, observers will feel they are protecting themselves from harm by learning from someone else's mistakes.

Overall, the common belief is that the victim got what they deserved, because in a justworld, good things happen to good people and bad things happen to bad people (van de Boss \& Maas, 2009). These ideas feed into how common it is for men to get away with harassment and how many people feel it is not an issue of real importance. The just-world hypothesis is an integral part of victim blaming as it shifts responsibility from the true perpetrator to the victim. Cognitive dissonance (discrepancy between one's attitude and belief which contributes to feelings of discomfort) plays a complementary role in this process. People can harmonize their beliefs that the men who harass have no responsibility in that context, because men are more important than women, and women who make mistakes get what they deserve. When people's ideas about the balance of power between victim and perpetrator are shifted, the harmony in cognition becomes erratic, and people thereby blame the victim to harmonize their beliefs and attitudes (Festinger, 1962).

According to Malle, Guglielmo, \& Monroe (2014), blame is both cognitive and social: "The cognitive, private side of blame is the process that leads to a judgement of blame; the social, public side is the act of expressing a blame judgement to another person” (p.148). In a cognitive sense, observers will respond to stimuli based on characteristic information processing aided by emotions; thus, the social blame occurs when there are roles and norms attributed to the situation. Individuals will tend to focus on their life experiences and education in any given situation in order to make sense of what happened and why, thereby instilling harmony back into their cognition (Festinger, 1962), without taking into regard the paradoxical nature of the justworld hypothesis and cognitive dissonance. The social cognitive side of victim blaming also includes self-blame.

Being a victim of street harassment and witnessing discrepancies of blame can often lead the victim to self-blame. Self-blame (or self-reproach) is an act of believing that one deserved to be harassed because of one's own actions (Janoff-Bulman, 1978). Instead of believing the perpetrator is to blame, it is often attributed that there was something one did to invoke the harassment insofar believing that one was wanting the attention, otherwise the harassment would have never taken place. This is a key example of how self-blame works and how it creates the discrepancy of who to blame and who to view as the victim (Macmillan, 2010). Thus, in a 'justworld', the victim blames her/himself because their actions triggered the attack.

Janoff-Bulman (1978) introduced "characterological” and "behavioural” self-blame (p. 1798) and how each affects the victim: characterological self-blame is associated with the belief in personal deservingness of negative outcomes, which involves attributions to a relatively non- 
modifiable source (one's character); behavioural refers to the victim needing to avoid future negative outcomes, which involves attributions to a modifiable source (one's behaviour). Thinking in this manner defies rational thinking because it removes the blame off the true perpetrator and shifts the blame onto the victim, as though the victim were the real perpetrator, which is far more deleterious to the victim. This is problematic as the victim should not feel the need to change behaviours in order to prevent a future attack. However, these discrepancies in cognition of beliefs and attitudes are invaluable to future research toward the reduction of such discrepancies and the changing of harmful biased perceptions.

\subsection{Bystander Effect}

The bystander effect refers to the observation that it is unlikely those witnessing an attack will stop an attack either by calling the police or intervening themselves to stop the attack (Latane \& Darley, 1968). Indeed, the stabbing of Kitty Genovese, which was witnessed by at least 38 people was not prevented, and because of the diffusion of responsibility, the witnesses watched the attack for more than a half hour without any intervention. It is concluded that each witness was aware of the other numerous witnesses, thus responsibility was shifted and nothing was done (Latane \& Darley, 1968).

The bystander effect is still prevalent globally, providing an insight to the curiosity of death and barbaric behaviour, and how the diffusion of responsibility functions in numerous cases. For example, in the case of the Richmond High School Incident in 2009, a crowd of over 20 people recorded and witnessed a gang-rape of a teenage girl, but no one intervened or called for help (Daily Mail, 2013). In the case of Ilan Halimi's torture and death in 2006 in France that lasted for months, several crowds gathered to watch the torture of the young man, but no one did anything until he was dead (Gurfinkiel, 2006).In the case of Shanda Sahrer's death in 1992, she was abducted from her home and the case reported several witnesses but no one stepped in to prevent her death (Lohr, 2014). These are all modern recorded cases of consequences of the bystander effect and how an abundance of help was available but no one made their selves available to help.

According to Bennett, Banyard, \& Garnhart (2013), the bystander's responses range from ignoring the situation, intervene to prevent the violence from escalating, call for help, or support the victim. Possible reasons why people do not intervene or become involved in some way is they may believe someone else will help, or they are afraid they themselves will be hurt. Social cognitions play a large role in the bystander effect because the observer must deem the observed behaviour as problematic before intervening. However, definitions of problematic behaviour in the context of gender-based violence are unclear because of individual differences in the acceptability of gender-based violence, adversarial views of sex, and rape-myth acceptance (Burt, 1980; Banyard, 2011).

Rape-myth acceptance, or acceptance of sexual harassment, is a form of victim blaming that reinforces and justifies holding myths about harassment to further blame the victims for their own victimization thereby justifying male harassment (Burt, 1980). These myths manifest in general statements such as 'she asked for it', 'what were you thinking walking alone at night?' or, 'it was just a compliment', or, 'you're too sensitive'. The observer will only help if feeling a responsibility to do so, but because of rape-myth acceptance and victim blaming there is a decreased likeliness to help, and an increase in perpetrator culpability Latane \& Darley, 1968). As years have gone by, there is some increase in education and likeliness of intervening as a result of community programs; however, the stigma still sticks as a result of the high frequency 
of victim blaming in communities (Banyard, Moynihan \& Plante, 2007) and the normalization of victim blaming and the bystander effect (Planty, 2002).

In the context of street harassment, there is usually little intervening but increased groupthink (tendency for groups to converge on an attitude), so to speak, insofar that others will join in with the street harassment. In groupthink, the group becomes concerned with only having a harmony among themselves instead of the group members being able to make rational decisions on their own (Janis, 1982). In other words, instead of one person in the group labelling the harassment as problematic, there is pressure to think alike and discouragement to act against the group consensus by intervening to stop the harassment.

So far we can see that street harassment is overwhelmingly about the perceptions of power and masculinity; if individual men in a group do not join in to harass women, their masculinity will be at question because gender stereotypes are ever-prevalent globally. So the bystander effect in this case can work in both not intervening but also joining in, in some capacity, be it encouraging the behaviour or simply watching. These behaviours are dangerous not only to the victims but also the harassers, to prove their masculinity to their cohorts. The bystander effect and the just-world hypothesis illustrate why street harassment and victim blaming is still relevant, because it is enabling the perpetrators to continue harassing. If witnesses watch, record, join in, or ignore acts of violence, it is safe to say they would do the same for street harassment.

\subsection{Context Effects}

The study done by Fairchild (2010) explored how context effects play a role in street harassment. The first study revealed how perceptions and experiences of harassment change depending on the context that will either influence fear or enjoyment of being catcalled. First, the results compiled how frequent whistles, leering, unwanted sexual attention, crude jokes, and seductive remarks were, finding that $34.5 \%$ of the participants, who were all heterosexual women, $(n=1277)$ reported harassment happening at least once a month. The clincher lies in the perceptions of street harassment and who is doing the harassing; by and large, the participants enjoyed the comments made by young, attractive men (between 18-30 years old), but felt more fear when the same comments were made by older and perceived unattractive men (40 and older).

It can be hypothesized that based on impression formation, or the schema that is formed of a person within a few seconds based on attributes such as gender and appearance and stereotypical beliefs (Fiske \& Taylor, 1991; Morin, 2014), attractiveness can be deemed generally as goodness, while unattractiveness can be generally deemed as maliciousness, which could explain why the women in the study perceived the harassers in different lights depending on the context. Nevertheless, the first study showed that women had more fear when they were by themselves at night, and when the harasser was a different race than they were. Fairchild (2010) also found that males who were tolerant of street harassment viewed it as an enjoyable experience for women. Perhaps those men did not want women to react in a negative way to the harassment if they were the ones doing it, or maybe those men in the study viewed street harassment as a compliment or self-esteem raiser meant to flatter the target, not offend or cause controversy. 


\subsection{Stereotypes}

It is often believed that only women are the victims of street harassment, but according to the Stop Street Harassment National Survey (2014), 25\% of victims are men. Further, the survey found that $50 \%$ of the individuals who took the survey had experienced street harassment by the age of 17; individuals who identified as LGBTQ+ (those not identifying as heterosexual) reported a higher frequency of street harassment than their heterosexual counterparts (57\% compared to 37\%). Neilson (2000) substantiated that white women and women of color experience the highest levels of street harassment, but it can affect anyone as illustrated above. What this means is that street harassment is a frequent situation that people of all races and gender deal with through no fault of their own.

It is a common belief that street harassment is an isolated and rare issue because of the infrequency of how often it is reported; on the contrary, the Stop Street Harassment National Survey (2014) found that $86 \%$ of women and $79 \%$ of men stated to be harassed on more than one occasion. Although it may not always be reported to the police, it does not disqualify how frequent street harassment is. This shows that the issue is not seen as serious by many, which could feed into why the victims tend not to report or talk about harassment. However, reports that are being published show that, there is a higher likeliness of changing perceptions of the pervasive nature of street harassment (Stop Street Harassment National Survey, 2014).

Street harassment has been stereotyped to be viewed as harassment only when older men of low socioeconomic standing are doing the harassing; however, men -and even a small percentage of women- regardless of socioeconomic status, have participated in street harassment (Stop Street Harassment National Survey, 2014). Further, not all street harassment is viewed as compliments by the perpetrator. For example, there are hateful and derogatory comments that are made by the perpetrator if, for instance, the target identified as gay or trans, they are more likely to be harassed differently than heterosexual women. Street harassment is most often done by men who are not known to the victim (Fairchild \& Rudman, 2008). This ties in with the aforementioned power dynamic, in which the men who are harassing the victims place their apparent biological power onto that victim, making him/her the object.

\section{Feminist Theories}

Street harassment is gender-based violence that is normalized in a power dynamic, which is used in everyday situations. These power dynamics are culturally normalized into societies to the extent that the topic itself was ignored for several decades, becoming virtually invisible. As more advocacy and awareness are seeping through mainstream media, the more individuals are faced with the pervasiveness of street harassment. Although it is a controversial topic, it is important to treat because the more awareness there is of street harassment and victim blaming prevalence and implications, the more conditioned bystanders may become to the fact that the victims are not the ones to blame.

Western culture behaves in an opprobrious manner when exposed to headlines or stories of sexual violence; the blame is shifted from the predator that caused the crime to the victim who is being blamed for a myriad of reasons. For most people, the lack of rationality is contributed to by the just-world hypothesis (van de Boss \& Maas, 2009). It is important to note that although there is a constant reference to western culture perpetuating street harassment and victim blaming, it is, in fact, found globally and is not only culturally-bound (Hollaback Ottawa, 2014). 


\subsection{Biological Determinism}

First, the theory that must be tackled is biological determinism, because it has a fundamental impact on gender schema formation. Biological determinism states that one's characteristics and traits are predominantly due to one's biological sex. In other words, a biological male will typically be expected to have masculine traits, where as a biological female will be expected to have feminine traits. These traits can be thought of as dualisms- a dichotomy in line with the two-sex model as follows for male and female characteristics respectively: active/passive, strong/weak, dominant/submissive, subject/object (Gannon, 2005). Masculine traits are thought of as more important or powerful, whereas the feminine traits are thought of as subordinate. Because of this dichotomy, gender is not thought of as a continuum wherein one person can identify as having both masculine and feminine traits; instead, society puts more emphasis and favourability towards those who have masculine or feminine traits. More specifically, masculine traits tend to be more favoured than feminine traits because femininity has too many negative connotations (Gannon, 2005).

Negative connotations such as being weak, emotional, and helpless are adjectives that are perceived unfavourable in this society, and are often discredited, which could lead to people perceiving that women (or effeminate men) should not only not be taken seriously but also not be seen on the same level as a man who harbours all of the 'correct' qualities. Now that I have outlined biological determinism I can frame it within feminist theories to highlight how social cognitive schemas, attitudes, and inferences affect gender formation.

\subsection{Objectification and Power Dynamics}

When women are discredited as being human beings, they are then seen as an object waiting to be acted upon by the male gaze. The gaze concept was originally coined by Lacan (1973), who described it as an awareness that one can be viewed as an object, a type of awareness associated with anxiety. However, Mulvey (1975) refined the term, which states that the woman is viewed for pleasure, thus she is seen as an object instead of a subject; in other words, a woman is sexually objectified so her worth is dependent on her appearance. Indeed, according to Saguy, Quinn, Dovidio, \& Pratto (2010) "sexual objectification is the viewing of people solely as de-personalized objects of desire instead of as individuals with complex personalities and desires/plans of their own” (p.180). Demeaning a person devalues them, which can perpetuate hate, and possibly violence. In this context, by objectifying a person, the harasser is contributing to a person's heightened awareness and anxiety, taking away basic human rights by perceiving that person as an object, and further, by taking away the right to a safe space.

Power dynamics and objectification are complementary as both contribute to how women tend to be perceived. Women are typically perceived as sexual objects for the interest of men, and as objects, women are taught not to oppose this, but instead, pine for it, because beauty and youth are powerful factors associated with sexual desire and tend to be the factors that contribute to how women are treated. Similarly, men are expected to adhere to their assigned gender roles and encouraged to objectify women, thus the gender power dynamic is a double-edged sword by which both genders are subject to being hurt. However, in terms of street harassment, any one individual can be the target of it, but as stated previously, women are disproportionately affected by it; street harassment genders the streets by distributing power and a gender hierarchy in the form of male dominance over female submissiveness (Davis, 2002). The main reason why street harassment is viewed as an invisible harm is because men tend to be at the top of the hierarchy 
and tend not to be the victims of harassment - or at least not to the same degree as women, rendering street harassment as less visible than if men were the typical victims.

Fairchild \& Rudman (2008) contend that society has rendered women to be looked at and touched for the entertainment of others (i.e., "benevolent sexism”, p. 340). Additionally, they are not seen as capable human beings, in fact, women are often defined by their gender, and often times not credited with being valuable and unique beings. By defining people by their gender, women are more susceptible to being victims of violence, and men are defined and limited by their gender roles which can contribute to them acting out in a violent or deviant manner. Similar to these power dynamics, oppression is the idea that a group of people are subjugated by an absolutely privileged group who dictates how the subjugated group should act and think. Women, overwhelmingly in media and in everyday life, are taught how to think and act especially about how to take comments from men, in which women are taught that any attention focusing on parts of their body is good attention and defines their worth (Davis, 2002).

\section{Personal Experiences}

To offer an alternative example on the normalization and complexity of street harassment, I compare my own personal experience to the aforementioned academic studies. I have dealt with street harassment since I was 13; in which the first instance I instantly felt an intrusion of my personal space. As time went on, I felt a confliction between thinking whether these comments or gestures were compliments or actual harassment, and in the last few years I have steadily thought these gestures, looks, comments, and leers were inappropriate and uncalled for. I have had men follow me, intrude in on my personal space, and objectify me, all of which I thought to myself that perhaps I deserved; I blamed myself for many of these instances because I thought that I could have prevented them by wearing something else or not walking alone at night or modifying my behaviour in some way to prevent harassment. In essence, instead of realizing that it was not my fault, and that the fault was with the people who chose to harass me, I blamed myself.

\subsection{Personal Experiences, Theory and Implications}

Many individuals who fall victim to harassment find themselves doing mental gymnastics, thinking it was their own fault that they were harassed, perhaps in accordance with the constant perpetuation of sexual objectification, gender roles, and the accompanying biased attitudes and beliefs (i.e., cognitive dissonance, just-world hypothesis, bystander effect) shown in the literature. When dissonance occurs from the conflict of attitudes or actions with beliefs held about street harassment (i.e., not doing anything to help victims even though we believe harassment is wrong), we can find mental harmony when we change our perceptions to re-align our beliefs with our actions by believing someone else will intervene to help and not intervening ourselves in a problematic situation (i.e., bystander effect), or simply by believing that the harassment was deserved (i.e., victim blaming).

To reiterate, street harassment is normalized globally, and its pervasiveness is everywhere, like a contagious disease - and its prevalence keeps growing. It is important to start a conversation on violence against women, men, trans, liminal (those who's orientation shifts through time), non-identifying individuals, and children, regardless of their apparent age, race, ethnicity, ability, class, sexual orientation, and dress. Starting these conversations and spreading awareness that street harassment - or any other type of harassment - is a relevant issue because harassment spreads and perpetuates violence. By spreading awareness, it is possible to encourage 
education on this issue to remind people of its pervasiveness and consequences to eventually decrease street harassment and violence.

\section{Conclusion}

Overall, patriarchal rule and the bombardment of media messages has conditioned us to think a certain way about the roles men and women are given and how they should assess and act in different situations. Cultures globally normalize abhorrent acts and blame victims, which seems backwards, but is very prevalent. Davis (2002) makes an invaluable point about the inconsequential views of street harassment: "First, because street harassment has been trivialized, women do not talk about it and are thus silenced" (p. 216). Further, Davis divulges that when women do want to open up about street harassment they are faced with doubt because it has become incredibly trivialized, thus no conversations are started. Lastly, when women repress the harm of street harassment, their perceptions can transform into self-blame, self-harm, or learning to accept the harassment as compliments (Davis, 2002).

This process of conditioning, normalization, silencing, biased perceptions, self-blame, harm, and violence reinforces the need to address the invisibility of street harassment and its effects. In such a manner, education, awareness, and further research is the best path to tackle this issue, to illuminate how unsafe it makes the victims feel, and how important it is for mass media to highlight the consequences and myths harassers perpetuate. Further, beliefs that leering, touching, and grabbing are forms of complimenting adds to the perpetuation of this process, another place where education would be essential. Indeed, if these beliefs, perceptions, and attitudes were compliments, they would not be seen as harassment and would not have such harmful consequences.

\section{References}

Banyard, V. L., Moynihan, M. M., \& Plante, E. G. (2007). Sexual violence prevention through bystander education: An experimental evaluation. Journal of Community Psychology, 35(4), 463-481.

Banyard, V. L. (2011). Who will help prevent sexual violence: Creating an ecological model of bystander intervention. Psychology of Violence, 1216-229.

Bennett, S., Banyard, V. L., \& Garnhart, L. (2014). To act or not to act, that is the question? Barriers and facilitators of bystander intervention. Journal of Interpersonal Violence, 29(3), 476-496.

Burt, M. R. (1980). Cultural myths and supports for rape. Journal of Personality and Social Psychology, 38(2), 217-230.

Daily Mail (2013). Two attackers in horrific homecoming dance gang rape sentenced to decades behind bars as victim forgives them because she 'deserves to be at peace.' Retrieved November 23, 2014 from http://www.dailymail.co.uk/news/article-2395396/Richmond-homecoming-dance-gangrape-attackers-sentenced-decades-bars.html

Darley, J. M., \& Latane, B. (1968). Bystander intervention in emergencies: Diffusion of responsibility. Journal of Personality and Social Psychology, 8(4), 377-383.

Davis, D. E. (2002). The harm that has no name: Street harassment, embodiment, and African American women. Gender Struggles: Practical Approaches to Contemporary Feminism, 214-225.

Fairchild, K., \& Rudman, L.A. (2008). Everyday stranger harassment and women's objectification. Social Justice Research, 21(3), 338-357.

Fairchild, K. (2010). Context effects on women's perceptions of stranger harassment. Sexuality \& Culture, 14(3), 191-216.

Festinger, L. (1962). A Theory of Cognitive Dissonance (Vol. 2). Stanford University Press.

Fiske, S. T., \& Taylor, S. E. (Eds.). (1991). Social cognition. NY: McGraw-Hill.

Foucault, M. (1977). Discipline and punish: The birth of the prison. Random House LLC.

Gannon, L. R. (2005). Women and aging: Transcending the myths. Routledge. 
Gurfinkiel, M. (2006). Tale of Torture and Murder Horrifies the Whole of France. Retrieved November 23, 2014, from http://www.nysun.com/foreign/tale-of-torture-and-murder-horrifies-thewhole/27948/

Janis, I. L. (1982). Groupthink: Psychological studies of policy decisions and fiascoes. $2^{\text {nd }}$ ed., p. 349. Boston: Houghton Miffin.

Janoff-Bulman, R. (1979). Characterological versus behavioral self-blame: inquiries into depression and rape. Journal of Personality and Social psychology, 37(10), 1798-1810.

Lacan, J. (1973). Four fundamental concepts of psychoanalysis. New York: WW Norton \& Co.

Lerner, M. J., \& Simmons, C. H. (1966). Observer's reaction to the" innocent victim": compassion or rejection? Journal of Personality and Social Psychology, 4(2), 203-210.

Lohr, D. (2014). Death of Innocence - The Murder of Young Shanda Sharer. Retrieved November 23, 2014 from http://www.crimelibrary.com/notorious_murders/young/shanda_sharer/1.html

Macmillan, R., Nierobisz, A., \& Welsh, S. (2000). Experiencing the streets: Harassment and perceptions of safety among women. Journal of Research in Crime and Delinquency, 37(3), 306-322.

Malle, B. F., Guglielmo, S., \& Monroe, A. E. (2014). A theory of blame. Psychological Inquiry, 25(2), 147-186.

Morin, A. (2014). Impression Formation \& Management [pdf]. Retrieved November 20, 2014 from http://www2.mtroyal.ab.ca/ amorin/Impression.pdf

Morin, A. (2014). Introduction to Social Cognition [pdf]. Retrieved November 20, 2014 from http://www2.mtroyal.ab.ca/ amorin/IntroSC.pdf

Mulvey, L. (1975). Visual pleasure and narrative cinema. Screen, 16(3), 6-18.

Nielsen, L.B. (2000). Situating legal consciousness: Experiences and attitudes of ordinary citizens about law and street harassment. Law \& Society Review, 334(4), 1055-1090.

Ottawa Hollaback! (2014). Top Ten Myths of Street Harassment. Retrieved November 23, 2014 from http://ottawa.ihollaback.org/top-ten-myths-of-street-harassment/

Planty, M. (2002).Third-party involvement in violent crime, 1993-99. US Department of Justice, Office of Justice Programs, Bureau of Justice Statistics.

Saguy, T., Quinn, D. M., Dovidio, J. F., \& Pratto, F. (2010). Interacting like a Body Objectification can Lead Women to narrow their Presence in Social Interactions. Psychological Science, 21(2), 178182.

Stop Street Harassment National Survey. (2014). Retrieved October 30, 2014 from http://www.stopstreetharassment.org/resources/statistics/sshstudies/

van den Bos, K., \& Maas, M. (2009). On the psychology of the belief in a just world: Exploring experiential and rationalistic paths to victim blaming. Personality and Social Psychology Bulletin, 35(12), 1567-1578. 